\title{
Malformations of the cranio-cervical junction: basilar impression
}

Gonzalo Bertullo $^{1}$, Viviana Cabrera ${ }^{2}$

\begin{abstract}
Cranio-cervical junction abnormalities are a rare combination of congenital or acquired malformations, linked to the occipital-cervical region that often leads to severe neurological disorders. There is a great clinical polymorphism and high variability in the natural history of these conditions, which determines therapeutic outcomes difficult to assess in a global sense. The authors of this report give an account of the case of malformative bone complex of cranio-cervical junction and carry out a non-systematic search of the literature.
\end{abstract}

Keywords: Cranio-cervical malformations; Basilar impression; Platybasia; High cord compression

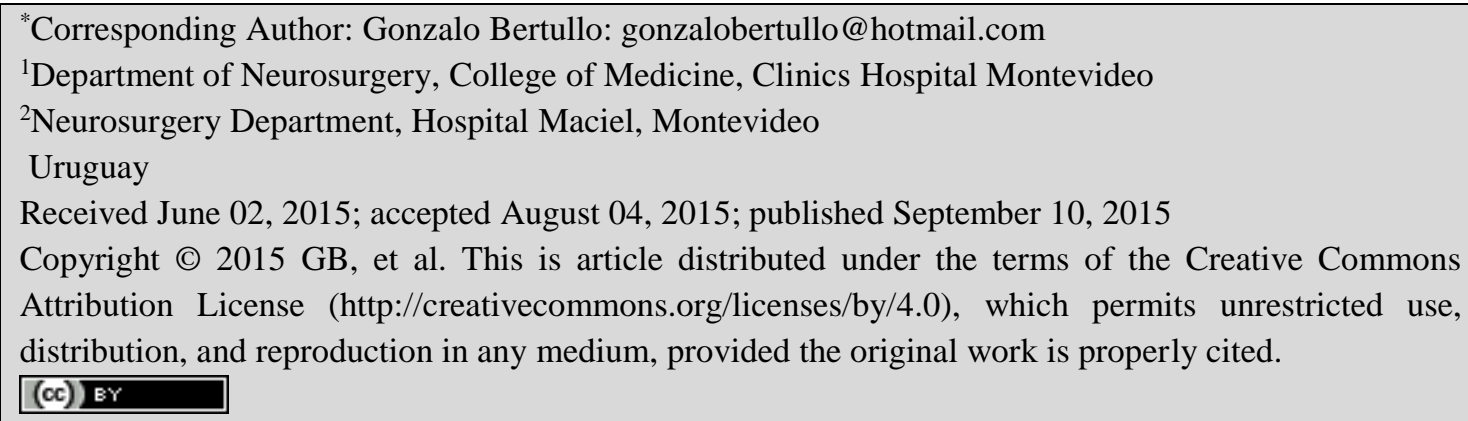

\section{Introduction}

Malformation or cranio-cervical dysplasia is known as a group of disorders in the endochondral portion of the occipital bone and of the first two cervical vertebrae, which as a result, is a variable set of malformations in the region.

Basilar Impression (BIM) is a deformity with a low incidence, but it represents the most common cause within the
Occipital-cervical deformations. There are two clinical forms: primary or congenital, and secondary or acquired. Several authors have described that symptomatic BIM becomes apparent when the odontoids extend $5 \mathrm{~mm}$ above the Chamberlain line (CHL). The CHL is an imaginary line joining the back of the hard palate to the rim of the foramen magnum (Fig.1 and 3). Moreover, the Boogaard's basal angle (BA) and the 
occipito-atloid-axoid angle junction, are other parameters that must be determined in the images of the craniocervical region, of which normal value is between $115-140^{\circ}$ and $125^{\circ}$, respectively (Fig. 1, 2 and 3).

In this paper, the case of a patient with malformative bone complex of the craniocervical region which led to a severe kinking of the brain stem (KBS), is reported. An analysis of the case, its imaging, and a non-systematic review of the literature is carried out.

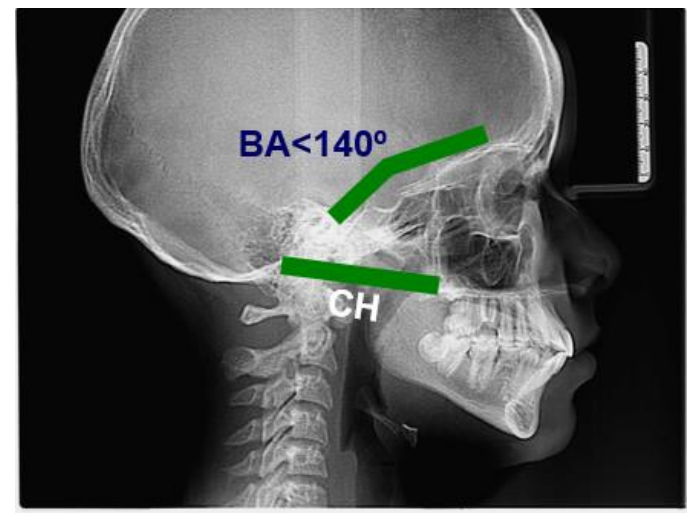

\section{Figure 1.}

$\mathbf{B A}=$ Intersection of a parallel line to the anterior base of the skull and other to clivus: Boogaard's basal angle $\left(115-140^{\circ}\right)$.

$\mathbf{C H}=$ Chamberlain line: hard palate to posterior rim of occipital foramen.

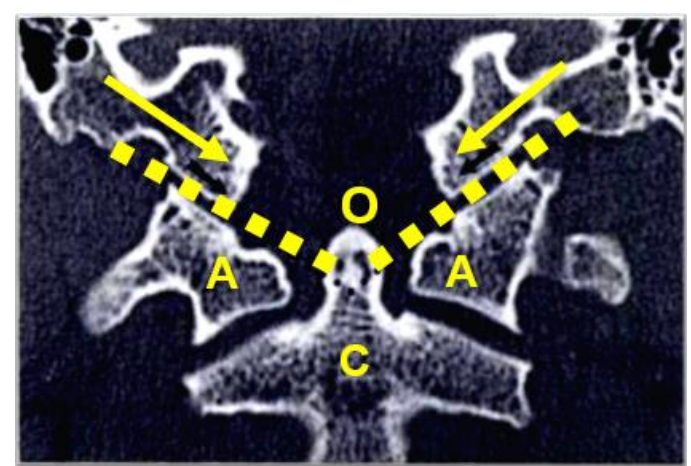

Figure 2.

Normal tomography of the skull (coronal view)
Points: Occipito-atloid-axoid angle junction Average angle with symmetrical condyles: $125^{\circ}$ Arrow: Occipital condyles

A: Atlas lateral masses

O: Odontoid apophysis of the axis

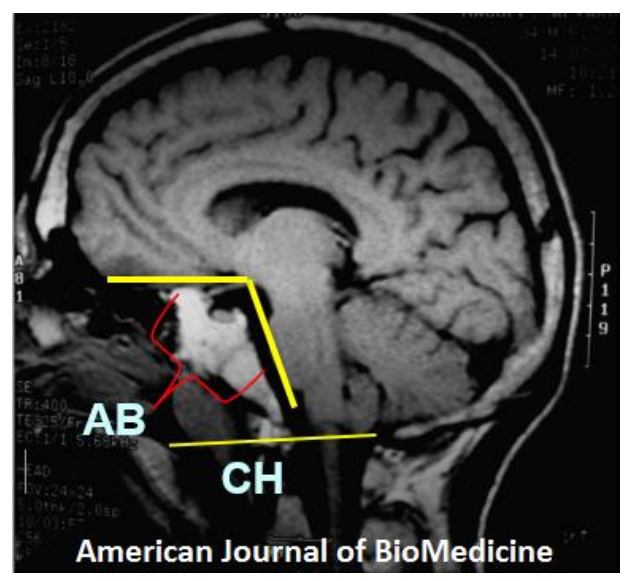

Figure 3.

Normal Magnetic Resonance (sagittal view).

$\mathbf{B A}$ and $\mathbf{C H}$ lines in resonance

\section{Clinical case}

Female, 43-year-old patient. 6-month progressive history of cervico-cephalea evolution, gait instability and difficulty holding objects. From the neurological examination a mild amblyopia with bilateral papilledema, hearing loss and bilateral cerebellar ataxia with the 4 members' hyperreflexia, was detected. General physical examination showed: short in stature, macrocephaly, hair growth on lower part of the nape, short neck prone to whiplash.

Plain radiographs (X-r), cranial computed tomography (CT) and magnetic resonance imaging (MRI) of 
head and cervical spine showed a malformative bone complex consisting of: platybasia (PTB), BIM, abnormal clivus (AC), odontoideum retrocess (OTR), hypoplasia of occipital condyles (HOC) and occipitalization of the atlas (OA) (Fig. 4, 5, 6, 7, 8, 9, 10).

These malformations determine severe brain stem compression with a secondary supratentorial hydrocephalus (Fig. 5, 6). During hospitalization the patient presented a rapid deterioration of consciousness, thus, an emergency ventricular peritoneal shunt (VPS) was performed. The patient's neurological evolution was unfavorable, later manifesting oculomotor alterations and lower cranial nerve dysfunction. The patient died a week after surgery.

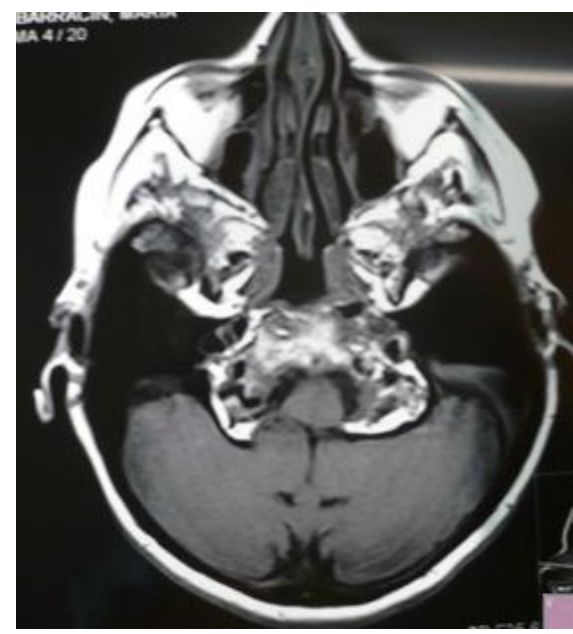

Figure 4.

MRI axial view, see severe MRI.

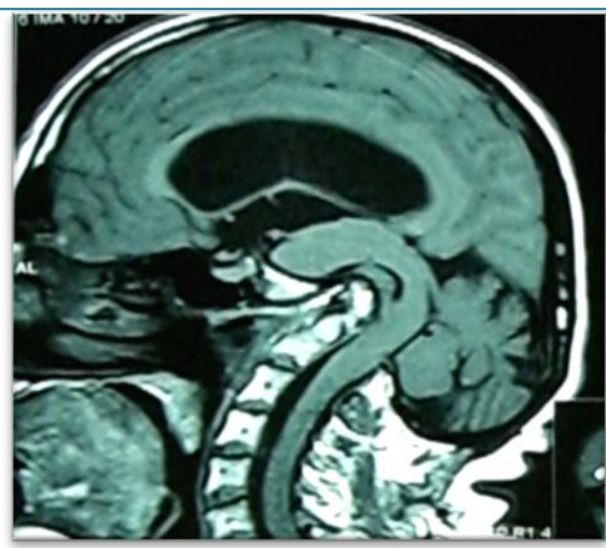

Figure 5.

KBS: Malformative complex is associated to a severe Kinking of the brain stem. BIM and severe PTB are detected.

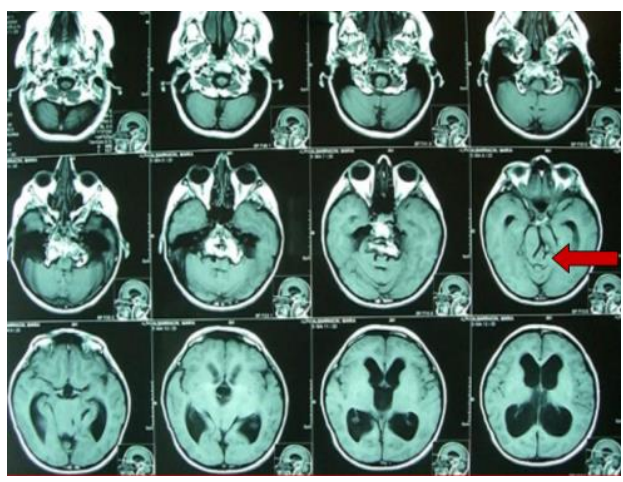

Figure 6.

Intracranial anatomical changes produced by BIM with consequent supratentorial hydrocephalus. Both vertebral arteries are displayed forming the basilar artery in a higher position than usual (arrow).

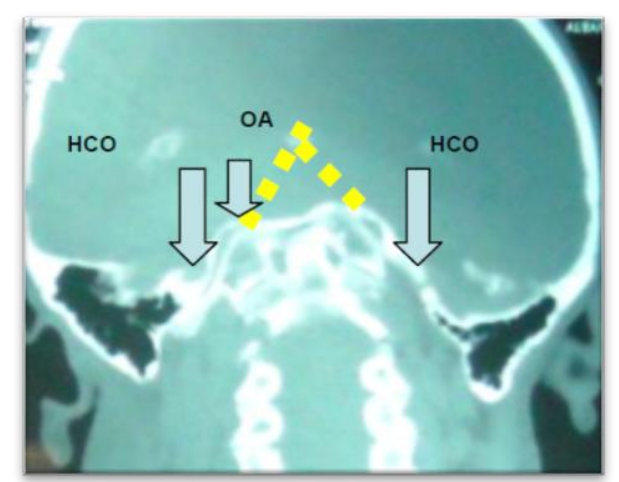

Figure 7.

HCO: Hypoplasia of the condyles. Points: Severe inversion of occipito-atloid-axiod angle junction product of BIM and HCO.

OA: Occipitalization of the atlas in CT coronal view. 


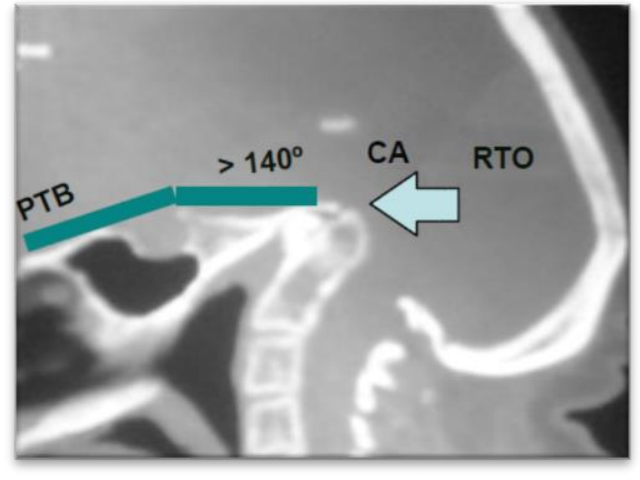

Figure 8.

PTB: Flattening of the skull base with a BA higher than $140^{\circ}$ : platybasia. Abnormal Clivus (CA): shortened and more horizontal.

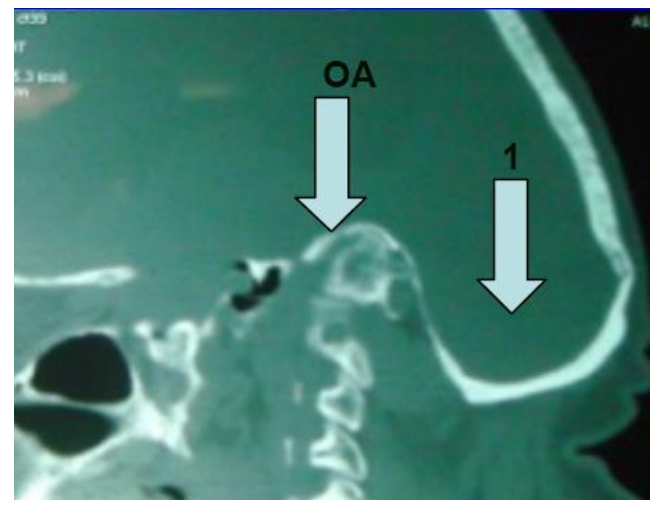

Figure 9.

"Convexobasia" cupular shape occipital is a feature of the BIM. Occipitalization of the atlas $(\mathrm{OA})$ is best appreciated.

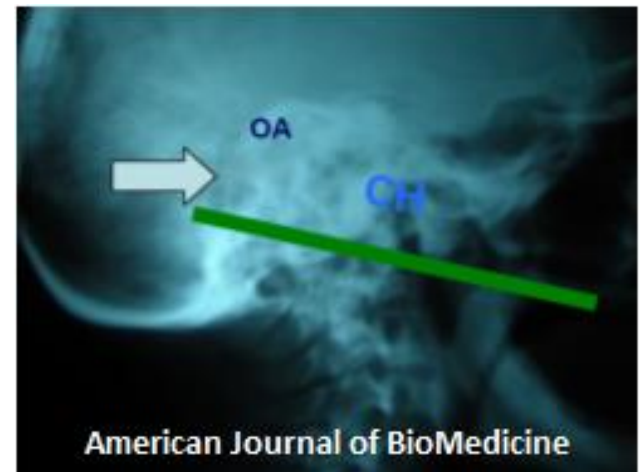

Figure 10.

IMB: See odontoideum rise above the $\mathrm{CH}$ line. OA: lateral continuity of the atlas and occipital is appreciated.

\section{Discussion}

Cranio-cervical junction abnormalities are congenital or acquired malformations of low incidence related to occipital-cervical junction that often determines serious neurological disorders. Its most frequent representative (BIM), is a bone ingrowth contour of the foramen magnum into the posterior fossa with reduced capacity, giving to the skull base a cupular shape opposite to the normal known as convexobasia (Fig. 9, 10) [1, 2].

This abnormal position is attributed to early synostosis of spheno occipital suture [1]. Its primary form is a congenital disease within the skeletal abnormalities of the cranio-cervical junction, which may or not be associated with other spinal defects (e.g., KlippelFeil syndrome) $[1,3]$.

On secondary or acquired form, cranial deformity arises from other synthetic diseases causing softening of bony structures. Among these the most common is rheumatoid polyarthritis and, in rarer cases, Paget's disease, osteomalacia, rickets, and osteogenesis imperfecta [1].

As typical characteristics, the foramen magnum is small, deformed, moved up 
within the cranial cavity, high clivus and the atlas is underdeveloped. It may be asymmetrical and is usually attached to the occipital bone (Fig. 8) [5, 6].

The odontoids and axis are projecting forward and upward from their normal position and invade the spinal canal (Fig. 5). The importance of this anomaly lies in the side effects generated on the Central Nervous System (CNS) [1-4].

By physical examination, the neck is short and tilted forward, hyperextended and with a stiff neck. There is limitation of cervical movements and constant pain in the region. Neurological manifestations are typical of a high spinal cord compression, such as loss of strength and spasticity, as well as cerebellar elements: gait instability, limb loss and sensory changes. The pressure effects on the brain stem can cause some cranial nerve involvement by direct compression $[5,6]$. Disorders are also evident in the circulation of cerebrospinal fluid (CSF) due to circulation obstruction at medullary bulb level, which is not uncommon to face an associated hydrocephalus (HCP) as a consequence (Fig. 6). This can occur for symptoms and signs of Intracranial Hypertension (ICH) and even papilledema.
They may be associated with other malformations that should always be ruled out by imaging $[5,6]$. There is considerable clinical variability, like in the natural history of these conditions. Consequently, severe deformity explains the neurological sign-symptom manifested in these patients. The deformity of the brain stem can be very variable, but it is present in all cases and is often what determines the severity of the medical condition. In the case analyzed, the patient had clear elements of compression brain stem with cranial nerves alteration, also associated with posterior fossa manifestations of cerebellar type (Fig. 5).

This, in turn, blocks the CSF passage and generates supratentorial hydrocephalus; hence patient's waking stage became deteriorated, was decompensated and required emergency CSF shunt (Fig. 6). In the RMI anatomical changes that distort the posterior fossa are evidenced with severe compression and Kinking of the brain stem (Fig. 4, 5, 6). Supratentorial hydrocephalus was secondary, condition by which the patient had to be operated performing an emergency VP shunt.

It must be emphasized that it had element of chronicity as a bilateral 
amblyopia was discovered evidencing fundus papilledema.

It is important to take into account that medullary bulb ischemia can affect the aggravation of the symptoms which led to the patient's death either by direct compression or by formation of both arterial and venous vessels that are distorted as can be seen in the picture (Fig. 6). In simple XR made to the patient, the severe hypoplasia of the occipital condyles can be seen in detail, the occipitalization of the atlas in the coronal view (Fig. 7). Figure 8 shows the flattening of the skull base forming a platybasia with abnormal, more horizontal and flattened clivus in the sagittal view.

In Figure 9, convexobasia is better evidenced, in cupular shape of the posterior fossa and the most severe occipitalization of the atlas. Finally in Figure 10, the odontoid process above the CHL is observed. In general, these are patients who do not live many years; it is rare to find, like in this case, someone exceeding four decades. The neurological manifestations usually become apparent in younger patients and they die due to this cause at an early age. This malformative disease has a high mortality as secondary injuries that occur are incompatible with life. This determines therapeutic outcomes which are difficult to assess in a global sense. While in some patients the treatment is surgical, in others, as this case evidenced, the severity of the malformation makes this option virtually impossible.

\section{Competing interests}

Authors declare that we have no competing interests.

\section{Authors Contributions}

All authors wrote, read and approved the final manuscript.

\section{References}

1. Smoker WR. Craniovertebral Junction: Normal Anatomy, craniometry and congenital anomalies. RadioGraphics 1994; 14:255-277.

2. Royo MB. Platibasia, impresion basilar, retroceso odontoideo y kinking del tronco cerebral, etiología común con la siringomielia, escoliosis, malformación de Arnold Chairi idiopáticas. REV NEUROL (Barc) 1996; 24(134):12411250.

3. Botelho RV, Neto EB, Patriota GC, et al. Basilar invagination: craniocervical instability treated with cervical traction and occipitocervical fixation. Case report. J Neurosurg Spine 2007; 7(4):444-9.

4. Goel A, Shah A. Atlantoaxial joint distraction as a treatment for basilar invagination: a report of an experience with 11 cases. Neurol India 2008; 56(2):144-50.

5. Goel A, Bhatjiwale M, Desai K. Basilar invagination: a study based on 
190 surgically treated cases. $J$

Neurosurg 1998; 88:962-8.

6. A Tassanawipas, S Mokkhavesa, S

Chatchavong, P Worawittayawong.

Magnetic Resonance imaging study of the craneocervical junction. Journal of

Orthopaedic Surgery 2005; 13(3):228-

231. 